Obere Extremität 2018 $\cdot 13: 237-245$

https://doi.org/10.1007/s11678-018-0479-8

Angenommen: 19. Juli 2018

(c) Der/die Autor(en) 2018

CrossMark
Eingegangen: 22. Mai 2018

Online publiziert: 21. August 2018

\author{
Fabian Plachel ${ }^{1,3} \cdot$ Marvin Minkus $^{1} \cdot$ Markus Scheibel $^{1,2}$ \\ ${ }^{\prime}$ Centrum für Muskuloskeletale Chirurgie, Charité-Universitätsmedizin, Berlin, Deutschland \\ ${ }^{2}$ Schulthess-Klinik, Zürich, Schweiz \\ ${ }^{3}$ Institut für Sehnen- und Knochenregeneration, Paracelsus Medizinische Privatuniversität, Salzburg, \\ Österreich
}

\section{Superiore Kapselrekonstruktion: Indikation und klinische Resultate}
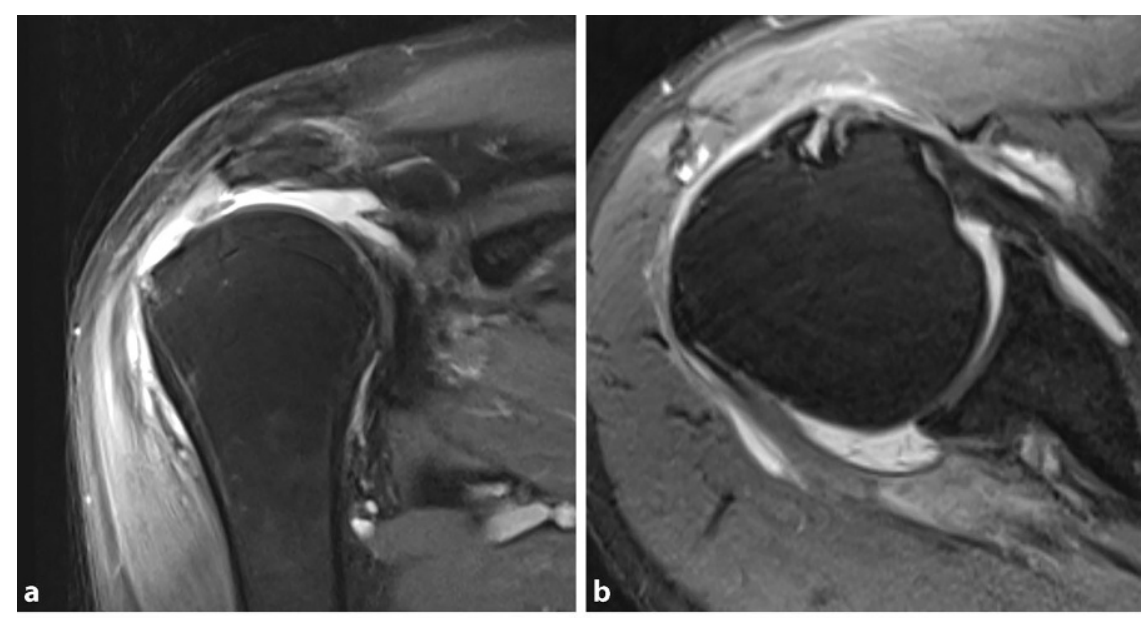

Läsionen der Rotatorenmanschette (RM) zeigen eine variable Ausprägung in Bezug auf die Pathogenese, Rupturmorphologie und daraus resultierenden funktionellen Einschränkungen. Mit einer Prävalenz von etwa $10-40 \%$ aller Rotatorenmanschettenrupturen (RMR) stellt die Massenruptur (mRMR) eine häufige Entität dar [1]. Diese ist definiert als (1) vollschichtige Ruptur mit einem anterosuperioren bzw. mediolateralen Defektaus$\mathrm{maß}$ von $>5 \mathrm{~cm}$ [6] bzw. (2) als Komplettruptur mit Beteiligung von $\geq 2$ Sehnen [9].

\section{) Die degenerative posterosuperiore Rotatorenman- schettenruptur stellt eine häufige Rupturmorphologie dar}

Am häufigsten beschrieben sind posterosuperiore RMR, welche in der Regel degenerativ bedingt sind und neben der Supraspinatussehne (SSP) den kranialen Anteil der Infraspinatussehne (ISP) bzw. den kompletten Footprint der ISP (sog. posterosuperiore $\mathrm{mRMR}$ ) miteinbeziehen. Sie unterscheiden sich v. a. hinsichtlich der Pathogenese (v.a. traumatisch bedingt) von den anterosuperioren RMR mit Beteiligung der Subscapularissehne (SSC; [28]).

Collin et al. [4] konnten in einer klinisch-radiologischen Arbeit zeigen, dass die individuelle Schulterfunktion wesentlich von der zugrunde liegenden Rupturmorphologie abhängt. Als
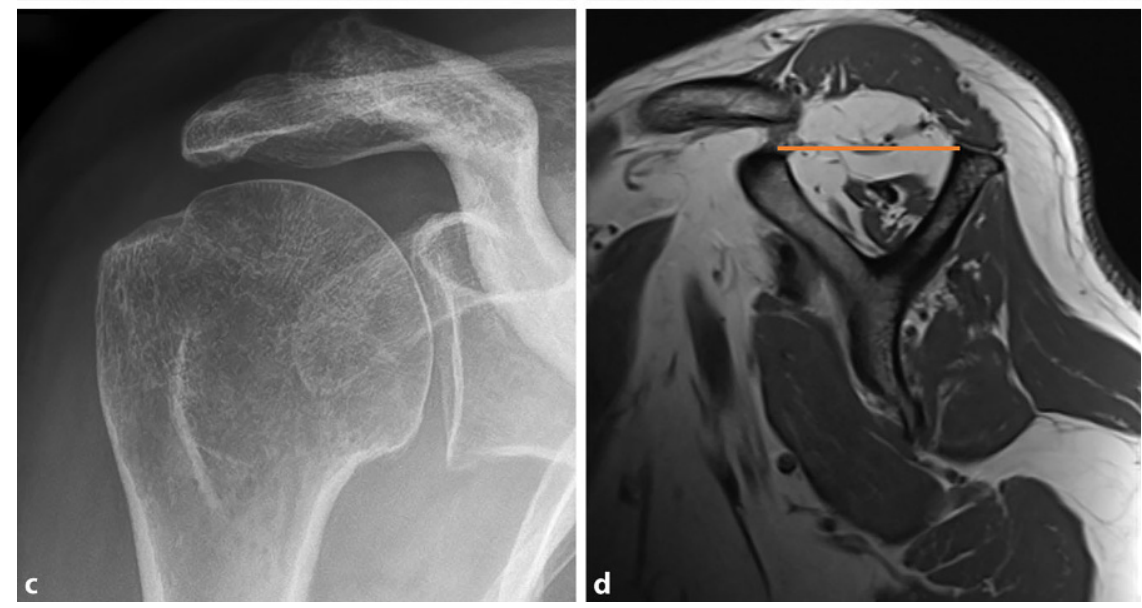

Abb. 1 \ Präoperative radiologische Diagnostik einer rechten Schulter. a In der koronaren Magnetresonanztomographie(MRT)-Aufnahme zeigt sich eine vollschichtige Supraspinatussehne(SSP)-Ruptur mit Sehnenretraktion bis supraglenoidal. b Das transversale Kräftepaar erscheint in der axialen Schicht intakt. c Nativ-radiologisch („true a.-p.-Aufnahme“) ist eine akromiohumerale Distanz von 5,0 mm zu messen, was einer Defektarthropathie Stadium 2 nach Hamada entspricht. $d$ Ein positives Tangentenzeichen (orange Linie) mit einer hochgradigen fettigen Degeneration des M. supraspinatus ist in der anguliert-sagittalen Aufnahme zu sehen 


\section{Übersicht}

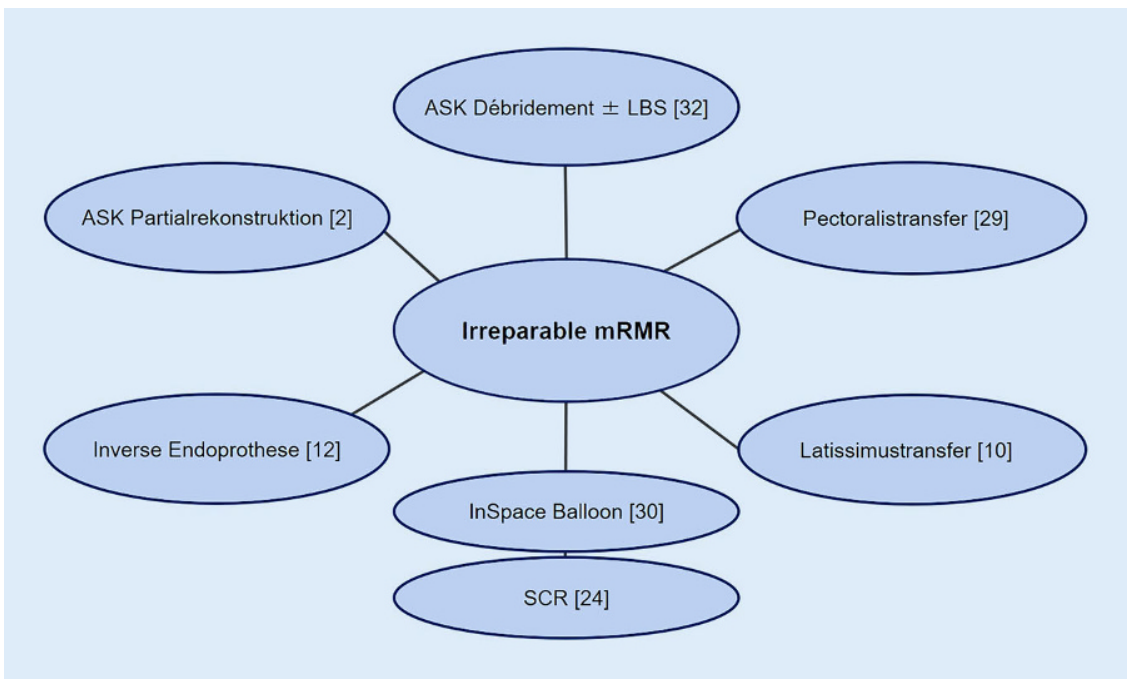

Abb. 2 - Operative Techniken zur Behandlung von irreparablen Rupturen der Rotatorenmanschette (RMR). ASK arthroskopisch, LBS lange Bizepssehne, SCR superiore Kapselrekonstruktion

weiteren Faktor einer defizitären Schulterfunktion postulierten die Autoren die progrediente fettige Muskeldegeneration, was wiederum die Bedeutung der zeitnahen Diagnostik mit entsprechender Therapie verdeutlicht. Primäres Ziel der operativen Therapie einer symptomatischen RMR ist die anatomische Rekonstruktion der betroffenen Sehnen, um dem natürlichen Progress entgegenzuwirken. Jedoch ist die Reparabilität und insbesondere die Heilungsrate nach arthroskopischer RM-Rekonstruktion u. a. von degenerativen Veränderungen, wie der Muskelatrophie bzw. -verfettung oder der Sehnenretraktion bzw. -länge, abhängig ([31]; - Abb. 1a). Als prognostisch ungünstig hat sich außerdem eine nativ-radiologisch gemessene akromiohumerale Distanz (AHD) von $<7 \mathrm{~mm}$ und ein magnetresonanztomographisch (MRT) detektiertes positives Tangentenzeichen gezeigt ([7, 33], - Abb. 1c, d).

Im Zweifel ist die Reparabilität bzw. Irreparabilität der RMR in Abhängigkeit patientenspezifischer Faktoren immer arthroskopisch zu evaluieren, da die mRMR gemäß Bedi et al. [1] nicht zwingend als in toto irreparabel zu werten ist und vice versa. Zusätzlich konnte gezeigt werden, dass ein struktureller Redefekt nicht zwangsläufig mit schlechteren klinischen Ergebnissen korreliert [3, 8]. Daraus resultiert auch die Empfehlung der Partialrekonstruktion bei Vorliegen einer partiell irreparablen posterosuperioren, anterosuperioren RMR oder globalen mRMR. Hierunter versteht man eine RMR mit irreparablen superioren Anteilen in Kombination mit reparablen Anteilen des transversalen Kräftepaares (ISP bzw. SSC). Ein Benefit der Partialrekonstruktion ist die Rebalancierung im Glenohumeralgelenk mit guten klinischen Ergebnissen im Kurzzeitverlauf [14].

Ist eine anatomische Rekonstruktion der RM aufgrund oben genannter Kriterien nicht mehr möglich, kommen neben bereits etablierten operativen Verfahren den modernen Techniken wie der Rekonstruktion der superioren Gelenkkapsel („superior capsular reconstruction“, SCR) eine entscheidende Rolle in der Behandlung der (partiell) irreparablen RMR zu (• Abb. 2; [2, 10, 12, 24, 25, 29, 30, 32]). Das zu präferierende Verfahren ist im Endeffekt individuell zu wählen. Im Folgenden soll dieser Artikel die anatomischen Grundlagen und klinischen Ergebnisse nach Anwendung der SCR in Abhängigkeit der Indikationsstellung beleuchten.

\section{Grundlagen}

Im Wesentlichen stellt sich die superiore Gelenkkapsel als dünne membranöse Struktur mit einer engen Lagebeziehung zur posterosuperioren RM (SSP bzw. ISP)

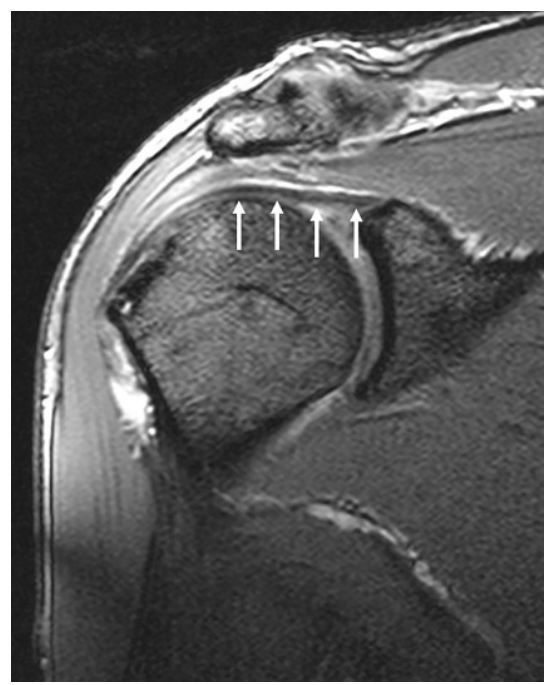

Abb. $3 \triangle$ Sagittale MRT-Aufnahme einer rechten Schulter (Pfeile Verlauf der superioren Gelenkkapsel von supraglenoidal nach humeral)

dar. In anatomischen Arbeiten konnte gezeigt werden, dass diese supraglenoidal entspringt, in ihrem Verlauf nach lateral v. a. durch das Lig. coracohumerale verstärkt wird und humeral am Tuberculum majus inseriert (•Abb.3). Die Ansatzzone deckt sich in etwa zu 30-61\% mit dem Footprint der posterosuperioren RM [26].

\section{》) Das Ausmaß der glenohume- ralen Dezentrierung hängt im Wesentlichen von der Integrität der Rotatorenmanschette ab}

Im Gegensatz zur posterosuperioren RM, welche im Verbund das Glenohumeralgelenk dynamisch durch zirkuläre Kompression stabilisiert, dient die superiore Gelenkkapsel als statischer Stabilisator. Mihata et al. [24] konnten in einer biomechanischen Arbeit zeigen, dass eine Defektsituation der superioren Stabilisatoren zu einer progredienten Translation des Humeruskopfes nach kranial führt. Klinisch lässt sich dieser pathophysiologische Vorgang durch das resultierende Ungleichgewicht im vertikalen Kräftepaar erklären. Das Ausmaß (dynamisch vs. statisch) der glenohumeralen Dezentrierung hängt im Weiteren von der Integrität der Rotatoren (transversales Kräftepaar; ISP bzw. SSC) ab (• Abb. 1b). 
Hier steht eine Anzeige.

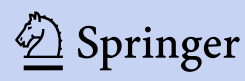


Liegt eine irreparable Defektsituation vor, ist man gezwungen, Verfahren heranzuziehen, welche das glenohumerale Rotationszentrum stabilisieren respektive wiederherstellen. Als Lösungsansatz wurde von Mihata et al. [24] ein Verfahren entwickelt, um die stabilisierende Funktion der nativen superioren Gelenkkapsel zu rekonstruieren, indem sie ein interossäres Transplantat (Fascia-lata[FL]-Autograft) zwischen Glenoid und Humerus implantierten. Dadurch konnte v. a. die superiore Translation des Humeruskopfes und der subakromiale Kontaktdruck signifikant reduziert werden, was klinisch der Progression der Defektarthropathie entgegenwirken soll.

In Folgearbeiten konnte gezeigt werden, dass eine simultane adäquate subakromiale Dekompression, ein dickeres Autograft und die Komplementierung der Rekonstruktion durch Zuhilfenahme von mehreren Seit-zu-Seit-Nähten zwischen dem nativen Sehnengewebe (ISP bzw. SSC) und dem Autograft die zentrierende Wirkung der Kapselrekonstruktion potenzieren [21-23].

Um die Entnahmemorbidität am Oberschenkel und in Folge die Operationszeit bei Verwendung eines Autografts zu reduzieren, wurde von Hirahara et al. [15] 2015 eine alternative Technik beschrieben, bei welcher ein humanes dermales Allograft (DA) verwendet wurde. Trotz oben genannter potentieller Vorteile und geeigneter Eigenschaften hinsichtlich Ausreißkraft zeigten erneut Mihata et al. [18], dass das Allograft dem Autograft biomechanisch unterlegen ist, was v. a. auf eine unphysiologische Elastizität des Allografts zurückgeführt wurde. Auch die Anwendung eines Xenografts als Interponat wurde beschrieben, jedoch gilt es hier erste Studien abzuwarten.

\section{Indikation}

Neben patientenspezifischen Faktoren, wie dem biologischen Alter, den führenden Beschwerden und dem funktionellen Anspruch, sind v.a. pathologiespezifische Faktoren wie die Rupturmorphologie und die Defektarthropathie als Entscheidungskriterien zur SCR heranzuziehen.

\section{Patientenspezifische Kriterien}

Historisch gesehen stellt die irreparable RMR in jüngeren Patienten mit erhöhtem Funktionsanspruch eine therapeutische Herausforderung dar. Aus diesem Grund wurde die SCR als Alternative $\mathrm{zu}$ etablierten nicht-anatomischen oder nicht-gelenkerhaltenden Verfahren wie dem Muskeltransfer oder der inversen Schulterendoprothetik v. a. für Patienten, welche zu jung, zu aktiv oder zu sportlich für eben diese Salvage-Prozeduren gelten, beschrieben. Jedoch konnten Untersuchungen zeigen, dass das chronologische Alter nicht als alleinstehendes Einoder Ausschlusskriterium herangezogen werden sollte. Bis zum jetzigen Zeitpunkt ist keine negative Korrelation zwischen dem Alter und dem subjektiven oder objektiven Ergebnis nach SCR nachgewiesen worden, was aufgrund der breiten Altersspanne von 27 bis 82 Jahren als substanziell angesehen werden kann [5, $16,17,19,20,27]$.

\section{》) Das chronologische Alter sollte nicht als alleinstehendes Ein- oder Ausschlusskriterium zur SCR herangezogen werden}

Unabhängig des Alters variieren insbesondere die Beschwerden und der individuelle Funktionsanspruch zum Zeitpunkt der präoperativen Vorstellung. Diesbezüglich konnten Mihata et al. [19] zeigen, dass ein minderer und im Umkehrschluss vermehrter beruflicher oder sportlicher Anspruch weder das klinische Ergebnis, noch die strukturelle Integrität negativ beeinflussen. Zusätzlich wurde der Residualfunktion der betroffenen Schulter oft eine besondere Bedeutung hinsichtlich der potentiellen Funktionszunahme eingeräumt. Hierbei ist v. a. die kompensierte Schulterfunktion mit einer Elevation über Schulterniveau von der nicht-kompensierten oder pseudoparalytischen Schulterfunktion $\mathrm{zu}$ differenzieren. Letztere ist definiert als Unfähigkeit, die betroffene Extremität über $90^{\circ}$ aktiv zu elevieren bei gleichzeitig vorliegender freier passiver Beweglichkeit.
Obere Extremität 2018 $\cdot 13: 237-245$

https://doi.org/10.1007/s11678-018-0479-8

(c) Der/die Autor(en) 2018

F. Plachel · M. Minkus · M. Scheibel

\section{Superiore}

Kapselrekonstruktion: Indikation und klinische Resultate

\section{Zusammenfassung}

Die optimale Therapie der irreparablen bzw. partiell reparablen Rotatorenmanschettenruptur wird weiterhin kontrovers diskutiert. In Abhängigkeit von patientenund pathologiespezifischen Faktoren bestehen sowohl konservative, als auch verschiedene operative Behandlungsoptionen. Das Verfahren der superioren Kapselrekonstruktion („,superior capsular reconstruction", SCR) mit Verwendung eines Auto- oder Allografts stellt hierbei einen relativ neuen Therapieansatz dar, dessen Indikationsgebiet bislang noch nicht abschließend geklärt ist. In diesem Artikel soll ein Überblick über die aktuelle Datenlage, die Indikationsstellung und erste klinische und strukturellen Ergebnisse der SCR gegeben werden.

Schlüsselwörter

Schultergelenk - Rotatorenmanschette . Irreparable Ruptur - Massenruptur .

Superiore Kapselrekonstruktion

\section{Superior capsular reconstruction: Indication and clinical results}

\section{Abstract}

The treatment of irreparable or partial reparable rotator cuff tendon tears is still discussed controversially. Depending on patient- and pathology-specific factors, conservative as well as different surgical treatment options exist. Superior capsular reconstruction (SCR) using an autograft or allograft represents a relatively novel surgical technique. However, the ideal indication for this procedure is still under debate. This review article provides an overview about the current literature concerning indications and early clinical and structural results of SCR.

\section{Keywords}

Shoulder joint - Rotator cuff · Irreparable tear - Massive tear - Superior capsule reconstruction 


\begin{tabular}{|c|c|c|c|c|c|c|c|c|c|}
\hline Studie & $\begin{array}{l}\text { Patienten } \\
\text { (n) }\end{array}$ & $\begin{array}{l}\text { Alter ( } \varnothing \\
\text { Jahre) }\end{array}$ & $\begin{array}{l}\text { Follow- } \\
\text { up ( } \varnothing \\
\text { Jahre) }\end{array}$ & Graft & $\begin{array}{l}\text { Klinik } \\
\text { (Punkt }\end{array}$ & & $\begin{array}{l}\text { AHD } \\
(\mathrm{mm})\end{array}$ & $\begin{array}{l}\text { Transplantat- } \\
\text { versagen } \\
(n, \%)\end{array}$ & $\begin{array}{l}\text { Komplikation } \\
\text { (\%) } \\
\text { Revision (\%) }\end{array}$ \\
\hline $\begin{array}{l}\text { Mihata et al. } \\
2013 \text { [20] }\end{array}$ & 24 & 65 & 34 & $\mathrm{FL}$ & $\begin{array}{l}\text { ASES: } \\
\text { VAS: }\end{array}$ & $\begin{array}{l}24 \rightarrow 93 \\
\text { n.e. }\end{array}$ & $4,6 \rightarrow 8,7$ & $1(4)$ & $\begin{array}{l}0 \\
0\end{array}$ \\
\hline $\begin{array}{l}\text { Mihata et al. } \\
2018 \text { [19] }\end{array}$ & 100 & 67 & 48 & $\mathrm{FL}$ & $\begin{array}{l}\text { ASES: } \\
\text { VAS: }\end{array}$ & $\begin{array}{l}36 \rightarrow 92 \\
\text { n.e. }\end{array}$ & n.e. & $5(5)$ & $\begin{array}{l}16 \\
0\end{array}$ \\
\hline $\begin{array}{l}\text { Hirahara et al. } \\
2017 \text { [16] }\end{array}$ & 8 & 61 & 32 & $\mathrm{DA}$ & $\begin{array}{l}\text { ASES: } \\
\text { VAS: }\end{array}$ & $\begin{array}{l}42 \rightarrow 87 \\
6,3 \rightarrow 0,4\end{array}$ & $4,5 \rightarrow 7,7$ & $2(40)$ & $\begin{array}{l}0 \\
13\end{array}$ \\
\hline $\begin{array}{l}\text { Denard et al. } \\
2017 \text { [5] }\end{array}$ & 59 & 62 & 18 & $\mathrm{DA}$ & $\begin{array}{l}\text { ASES: } \\
\text { VAS: }\end{array}$ & $\begin{array}{l}44 \rightarrow 78 \\
5,8 \rightarrow 1,7\end{array}$ & $6,6 \rightarrow 6,7$ & $11(55)$ & $\begin{array}{l}7 \\
19\end{array}$ \\
\hline $\begin{array}{l}\text { Pennington } \\
\text { et al. } 2018 \text { [27] }\end{array}$ & 86 & 59 & 12 & $\mathrm{DA}$ & $\begin{array}{l}\text { ASES: } \\
\text { VAS: }\end{array}$ & $\begin{array}{l}52 \rightarrow 82 \\
4,0 \rightarrow 1,5\end{array}$ & $7,1 \rightarrow 9,7$ & n.e. & $\begin{array}{l}0 \\
2\end{array}$ \\
\hline $\begin{array}{l}\text { Lee und Min } \\
2018 \text { [17] }\end{array}$ & 36 & 61 & 25 & $\mathrm{FL}, \mathrm{DA}$ & $\begin{array}{l}\text { ASES: } \\
\text { VAS: }\end{array}$ & $\begin{array}{l}50 \rightarrow 84 \\
5,8 \rightarrow 1,6\end{array}$ & $4,9 \rightarrow 8,0$ & $13(36)$ & $\begin{array}{l}0 \\
36\end{array}$ \\
\hline
\end{tabular}

Tab. 2 Indikations- und Operationsempfehlungen

\begin{tabular}{|c|c|c|}
\hline $\begin{array}{l}\text { Pathologiespezifische } \\
\text { Kriterien }\end{array}$ & $\begin{array}{l}\text { Patientenspezifische } \\
\text { Kriterien }\end{array}$ & $\begin{array}{l}\text { Operationsspezifische } \\
\text { Kriterien }\end{array}$ \\
\hline Rupturmorphologie & Alter & Transplantatdicke \\
\hline \multirow{2}{*}{$\begin{array}{l}\text { Partiell irreparable antero- bzw. } \\
\text { posterosuperiore RMR oder } \\
\text { globale mRMR }\end{array}$} & Ohne Einfluss & $\mathrm{FL}:>6 \mathrm{~mm}$ \\
\hline & Schulterfunktion & $\mathrm{DA}:>3 \mathrm{~mm}$ \\
\hline Defektarthropathie [13] & $\begin{array}{l}\text { Kompensiert und dekom- } \\
\text { pensiert }\end{array}$ & Partialrekonstruktion \\
\hline Stadium $1+2$ & Funktionsanspruch & $\begin{array}{l}\text { Wenn möglich unbedingt } \\
\text { erwägen }\end{array}$ \\
\hline Fettige Muskeldegeneration [11] & Ohne Einfluss & $\begin{array}{l}\text { Adaption von Graft zu nativem } \\
\text { Gewebe }\end{array}$ \\
\hline SSC + ISP: Grad $1+2$ & & Posterior $>$ anterior \\
\hline \multicolumn{3}{|c|}{$\begin{array}{l}\text { RMR Rotatorenmanschettenruptur, mRMR Massenruptur, SSC Subscapularissehne, ISP Infraspina- } \\
\text { tussehne, FL Fascia-lata-Autograft, DA humanes dermales Allograft }\end{array}$} \\
\hline
\end{tabular}

Die „wahre“ funktionelle Pseudoparalyse ist zwingend von einer „falschen“ schmerzassoziierten Pseudoparalyse (z.B. mittels Infiltrationstest) zu unterscheiden. Hinsichtlich der präoperativen, teilweise stark variierenden funktionellen Einschränkung wurde kein Einfluss auf das finale Ergebnis nach SCR beschrieben. Mihata et al. [20] konnten zeigen, dass eine pseudoparalytische Schulterfunktion durch die SCR in fast allen Fällen reversibel war. Diese Beobachtung wurde auch von Pennington et al. [27] beschrieben und zum einen auf die signifikante Schmerzreduktion und zum anderen auf die glenohumerale Rezentrierung zurückgeführt. Jedoch ist die Interpretation dieser Daten limitiert, da keine Aussage bzw. Unterscheidung in
Bezug auf die Pathogenese der Pseudoparalyse vorgenommen wurde. Etwaige Voroperationen scheinen keinen negativen Einfluss auf die SCR-Versorgung zu haben.

\section{Pathologiespezifische Kriterien}

\section{Rupturmorphologie}

Im Vordergrund der Indikationsstellung auf Basis pathologiespezifischer Kriterien steht die exakte Beurteilung der Rupturmorphologie. In der Pionierarbeit von Mihata et al. [20] aus dem Jahr 2013 wurden alle Patienten mit einer SCR versorgt, welche eine arthroskopisch verifizierte, partiell irreparable RMR aufwiesen. In ca. $58 \%$ der Fälle lag eine posterosuperiore RMR vor, wenngleich keine Aus- kunft zum Ausmaß der ISP-Schädigung gemacht wurde. In den restlichen $42 \%$ wurde eine posterosuperiore $\mathrm{mRMR}$ mit Beteiligung des kranialen SSC beschrieben.

Zusammenfassend war bei allen $\mathrm{Pa}$ tienten eine irreparable SSP-Ruptur mit simultaner reparabler ISP- und SSCLäsion evident. Letztere Sehnendefekte wurden zu $100 \%$ in Form einer Partialrekonstruktion adressiert. Im Gegensatz dazu wurden von Denard et al. [5] und Pennington et al. [27] alle Patienten mit einer irreparablen posterosuperioren mRMR eingeschlossen. Sowohl SSP als auch ISP waren in beiden Patientenkollektiven komplett rupturiert und wurden arthroskopisch als nicht anatomisch rekonstruierbar eingestuft. Lediglich die kraniale Läsion der SSC (56\%) wurde jeweils mittels Fadenankertechnik adressiert [5].

Die pathomorphologischen Einschlusskriterien in der Arbeit von Lee und Min [17] waren sehr heterogen und reichten von der isolierten irreparablen SSP-Ruptur (6\%) über die irreparable postero- (32\%) bzw. partiell irreparable anterosuperiore $(6 \%)$ RMR bis hin zur mRMR mit Beteiligung von 3 Sehnen $(56 \%)$. Auch in diesem Kollektiv wurde ausschließlich die kraniale SSC-Ruptur rekonstruiert.

\section{Muskeldegeneration}

Während fortgeschrittene degenerative Veränderungen an Muskel und Sehne ei- 


\section{Übersicht}
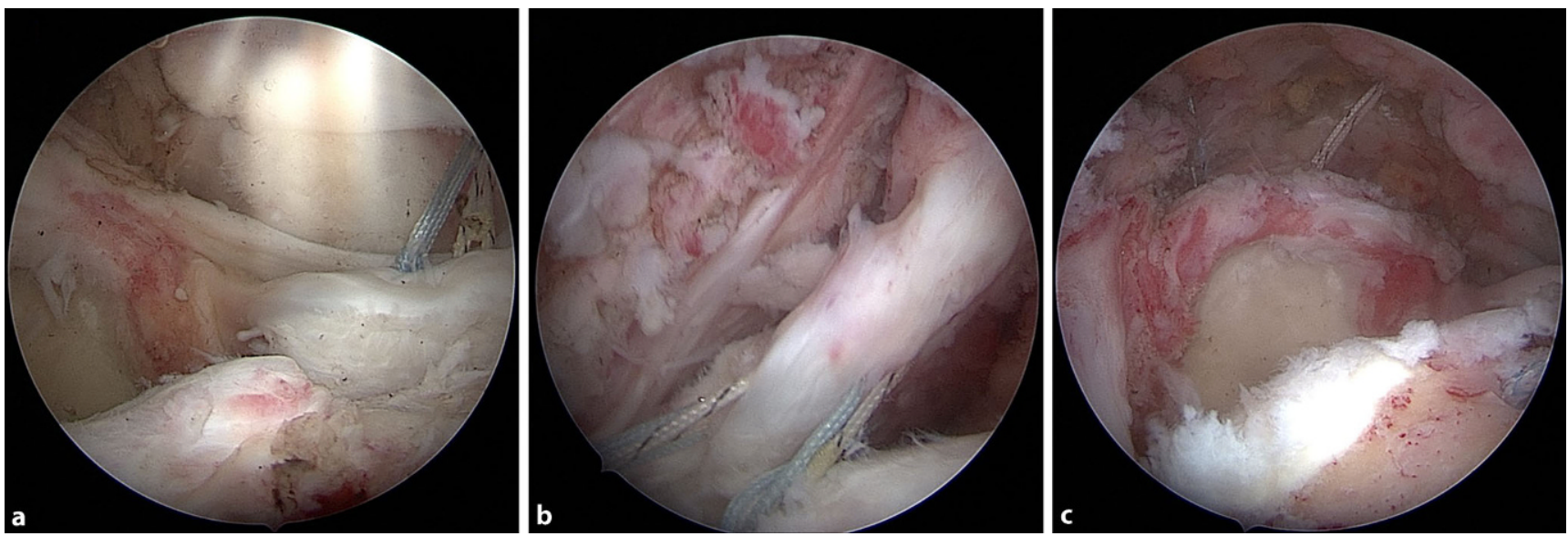

Abb. $4 \Delta$ Arthroskopie einer rechten Schulter mit radiologisch verifizierter partiell irreparabler Rotatorenmanschettenruptur (RMR) mit Beteiligung der kranialen Infraspinatussehne (ISP) und Subscapularissehne (SSC). Blick von lateral: a Die kraniale SSC-Läsion wurde mittels Fadenanker rekonstruiert, gleichzeitig die lange Bizepssehne (LBS) tenodesiert. b Rekonstruktion der kranialen ISP in doppelter Matratzennahttechnik. c Vorbereitung zur "superior capsular reconstruction“ (SCR). Die SSP zeigt sich irreparabel bis glenoidal retrahiert

ne angemessene Rekonstruktion der SSP negativ beeinflussen bzw. unmöglich machen, ist eine suffiziente Reinsertion von ISP bzw. SSC aufgrund der günstigeren Relation von Muskelvolumen zu Sehnenlänge auch bei einer höhergradigen Muskeldegeneration zu erzielen [2]. Wesentliche Gemeinsamkeit aller vorliegenden Arbeiten in Bezug auf die Pathoanatomie der RM war die fortgeschrittene Degeneration des M. supraspinatus, welche überwiegend als Grad 3 bzw. 4 nach Goutallier et al. [11] klassifiziert wurde. Eine wesentlich größere Spannbreite ist im Falle der transversalen Rotatoren (ISP bzw. SSC) zu vernehmen, welche das gesamte Spektrum der Muskelverfettung umfasste.

\section{Defektarthropathie}

Als relatives Ausschlusskriterium wurde in allen Arbeiten die endgradige Defektarthropathie mit Azetabularisierung und beginnender Humeruskopfdestruktion (Stadium 5 nach Hamada et al. [13]) angesehen.

\section{) Eine höhergradige Defektarthropathie wird als relatives Ausschlusskriterium angesehen}

Auch wenn in der Arbeit von Mihata et al. [20] Patienten mit „manuell nicht reponierbarem, nach kranial dezentrier- ten Humeruskopf " ausgeschlossen wurden, ist zu sehen, dass auch Fälle mit einer höhergradigen (bis Stadium 4 [13]) Defektarthropathie inkludiert wurden. In Anbetracht der Wertigkeit der SCR wurden aber zum Großteil (80-92\%) Patienten mit einer niedriggradigen Defektarthropathie (Stadium 1 und 2 [13]) eingeschlossen.

\section{Klinische Ergebnisse}

Zum jetzigen Zeitpunkt finden sich insgesamt 6 klinische Studien mit einer Mindestnachbeobachtung von 12 Monaten nach arthroskopischer SCR $[5,16,17,19$, $20,27]$. Bei der Interpretation der Daten müssen v.a. die Indikationsstellung und die Transplantatwahl berücksichtigt werden.

Alle Autoren berichteten übereinstimmend über eine im Durchschnitt signifikante klinische Verbesserung in Bezug auf die Schulterfunktion und die Schmerzintensität im Kurzzeitverlauf [5, 16, 20, 27]. Diese Zunahme spiegelte sich auch anhand aller erhobenen Bewertungsbögen wieder (• Tab. 1). Mihata et al. [20] zeigten, dass es nach SCR mittels Autograft sowohl zu einer signifikanten Zunahme der aktiven Schulterbeweglichkeit in allen Ebenen (Elevation $+64^{\circ}$, Außenrotation $+14^{\circ}$ und Innenrotation +2 Lendenwirbelkörper), als auch zu einer signifikanten Kraftzunahme der betroffenen Schul- ter gekommen ist, wenngleich im MRT keine wesentliche $\mathrm{Zu}$ - oder Abnahme der vorliegenden Muskelatrophie nachzuweisen war. Vergleichbare klinische Ergebnisse wurde auch von Pennington et al. [27] nach Verwendung eines Allografts beschrieben.

Eine Diskrepanz besteht in der Veränderung der AHD von prä- zu postoperativ, welche als radiologischer Parameter zur Beurteilung der glenohumeralen Zentrierung herangezogen wurde. Während es in den meisten Fällen zu einer signifikanten Zunahme direkt postoperativ gekommen ist, berichteten Denard et al. [5] lediglich von einer geringfügigen Vergrößerung mit einer Tendenz zur Abnahme über die Zeit. Ebenso zeigten sich essentielle Unterschiede in der strukturellen Transplantatintegrität, welche postoperativ im MRT evaluiert wurde.

Mihata et al. [19] wiesen eine FLGraftruptur ohne Angabe zur Lokalisation in lediglich $5 \%$ der Fälle bzw. ein Versagen der ISP-Rekonstruktion in $13 \%$ der Fälle nach. Demgegenüber steht eine Versagensrate von ca. 40-60\% nach Verwendung eines DA-Allografts [5, 16, 17]. Am häufigsten wurden hier humerale Komplettausrisse beschrieben, gefolgt von glenoidalen Defekten und intrasubstanziellen Rupturen.

Alle Autoren kamen zur Erkenntnis, dass ein substanzieller Schaden am Graft bzw. der initialen RM-Partialrekon- 

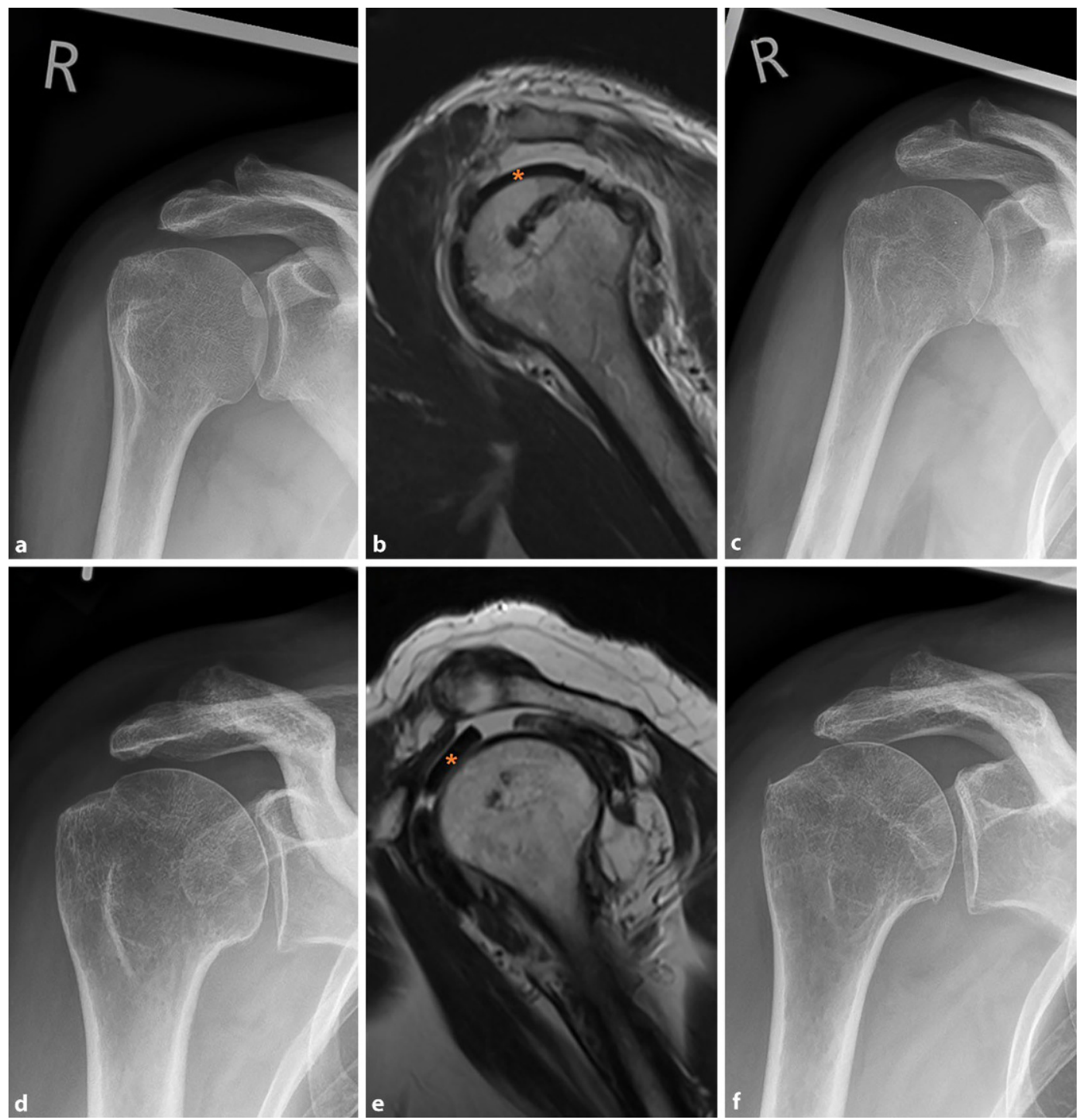

Abb. 5a-f $\triangleleft$ Rechte Schulter: a Präoperativ zeigt sich eine Defektarthropathie Stadium 2 mit einer AHD von $5,9 \mathrm{~mm}$. b Parasagittale MRT-Aufnahme 6 Wochen nach "superior capsular reconstruction" (SCR) mittels Allograft (oranger Stern) und suffizienter Adaption zum nativen Sehnengewebe des ISP(Infraspinatussehne)Gewebes bzw. zum anterioren Intervallgewebe. c Konventionell radiologische Verlaufskontrolle 12 Monate postoperativ mit glenohumeraler Rezentrierung und einer akromiohumeralen Dis$\operatorname{tanz}$ (AHD) von $10,5 \mathrm{~mm}$. d Niedriggradige Defektarthropathie Stadium 2 mit einer AHD von 5,0 mm. e Im MRT 6 Wochen nach SCR bestätigt sich die insuffiziente Reinsertion von Allograft (oranger Stern) zum vitalen ISP-Gewebe trotz intraoperativer ISP-Rekonstruktion. 6 6 Monate nach SCR zeigt sich eine persistierende glenohumerale Dezentrierung mit einer AHD von 5,2 mm

struktion (Komplettruptur bzw. „failure in continuity") einerseits zu signifikant schlechteren klinischen Ergebnissen und andererseits zu einer inadäquaten glenohumeralen Rezentrierung beigetragen hat. Patienten mit im MRT verifiziertem Transplantatversagen kombiniert mit postoperativer Schmerzprogression und regredienter Schulterfunktion wurden z.T. mittels inverser Schulterendoprothese oder einer Re-SCR revidiert $[5,16,27]$. Zusätzlich sind auch SCRunabhängige Komplikationen beschrieben. Hierzu zählen tiefe glenohumerale Infektionen, Ausrisse der verwendeten Fadenanker oder postoperative Schultersteifen, welche entsprechend mittels arthroskopischem Débridement, Im- plantatentfernung oder Kapselrelease therapiert werden konnten $[5,19]$.

\section{Indikations- und operationsspezi- fische Ergebnisse}

Auch wenn der direkte Vergleich zwischen den Arbeiten von Mihata et al. [20] und den amerikanischen Studien v. a. durch die Transplantatwahl erschwert ist, zeigt sich ein deutlicher Unterschied in Bezug auf die Indikationsstellung [5, 27]. Während Mihata et al. [20] die SCR als Additivverfahren zur Partialrekonstruktion der transversalen Rotatoren verwendeten (• Abb. 4), wurde in den Arbeiten von Denard et al. [5] und Pennington et al. [27] die SCR zur alleinigen Thera- pie der irreparablen posterosuperioren mRMR angewandt.

\section{》) Die SCR ermöglicht nach aktueller Datenlage zufriedenstellende klinische Ergebnisse}

In einer kürzlich publizierten Arbeit wurde die Bedeutung der posterioren RM (ISP) aufgezeigt [17]. Es konnte gezeigt werden, dass die ISP-Rekonstruktion signifikant die strukturelle Integrität der SCR und in Folge die glenohumerale Rezentrierung beeinflusst. Zusätzlich korrelierte die suffiziente Adaption von Graft und nativem Sehnengewebe mit- 
tels Seit-zu-Seit-Nähten positiv mit dem klinischen Ergebnis (• Abb. 5). Auch dem anterioren Anteil des transversalen Kräftepaares wurde eine wichtige $\mathrm{Be}$ deutung zugeschrieben, indem der Grad der SSC-Atrophie als negativer Prädiktor für ein strukturelles SCR-Versagen ausfindig gemacht wurde (GoutallierStadium 2,2 vs. 0,6 [11,5]). Zwischen den Arbeitsgruppen besteht Einigkeit darüber, dass die SCR v.a. Patienten mit niedriggradiger Defektarthropathie (Stadium 1 und 2 [13]) vorbehalten sein sollte. Denard et al. [5] konnten zeigen, dass die Erfolgsrate nach SCR in Abhängigkeit der Defektarthropathie von $75 \%$ bei Patienten im Stadium 1 bzw. 2 auf $44 \%$ bei statisch dezentriertem Schultergelenk (Stadium 3 bzw. 4) abnimmt.

Neben den potentiellen biologischen Vorteilen des Autografts zeigte sich v.a. hinsichtlich der $\mathrm{zu}$ erreichenden Transplantatdicke ein wesentlicher Unterschied zum Allograft. So ist mittels FL-Autograft durch Doppelung eine annähernd anatomische Dicke von bis $\mathrm{zu} 8 \mathrm{~mm}$ zu erreichen, wohingegen das Allograft bei maximal $3 \mathrm{~mm}$ limitiert ist. In beiden Fällen zeigen die aktuellen Ergebnisse, dass auf ein stärkeres, anatomiegetreues Transplantat (FL, $>6 \mathrm{~mm}$; DA, $>3 \mathrm{~mm}$ ) zurückgegriffen werden sollte $[5,17]$. - Tab. 2 gibt die wichtigsten, evidenzbasierten Empfehlungen und unsere eigenen Erfahrungen wieder.

\section{Fazit für die Praxis}

- Es sind v. a. pathologiespezifische

Kriterien entscheidend, ob eine

SCR indiziert ist oder auf andere, etablierte Verfahren zurückgegriffen werden sollte.

- Es besteht Einigkeit darüber, dass eine klinisch symptomatische irreparable oder partiell reparable posterosuperiore RMR mit niedriggradiger Defektarthropathie aktuell als Hauptindikation zu werten ist. Vor allem die Reparabilität der transversalen Rotatoren scheint einen positiven Effekt auf die klinischen und strukturellen Ergebnisse zu haben.
- Die SCR ermöglicht nach aktueller Datenlage zufriedenstellende klinische Ergebnisse mit v.a. einer deutlichen Schmerzreduktion, aber auch einer funktionellen Verbesserung.

- Nichtsdestotrotz müssen weitere Studien, v. a. Langzeituntersuchungen, abgewartet werden, um den Effekt einer dauerhaften Rezentrierung bzw. die Graftintegrität (biologische Heilung) unter Berücksichtigung der hohen Kosten des Verfahrens abschließend beurteilen zu können.

\section{Korrespondenzadresse}

\section{Dr. F. Plachel}

Centrum für Muskuloskeletale Chirurgie,

Charité-Universitätsmedizin

Augustenburger Platz 1, 13353 Berlin,

Deutschland

Fabian.Plachel@pmu.ac.at

Funding. Open access funding provided by Paracelsus Medical University.

\section{Einhaltung ethischer Richtlinien}

Interessenkonflikt. F. Plachel, M. Minkus und M. Scheibel geben an, dass kein Interessenkonflikt besteht.

Dieser Beitrag beinhaltet keine von den Autoren durchgeführten Studien an Menschen oder Tieren.

Open Access Dieser Artikel wird unter der Creative Commons Namensnennung 4.0 International Lizenz (http://creativecommons.org/licenses/by/4.0/deed. de) veröffentlicht, welche die Nutzung, Vervielfältigung, Bearbeitung, Verbreitung und Wiedergabe in jeglichem Medium und Format erlaubt, sofern Sie den/die ursprünglichen Autor(en) und die Quelle ordnungsgemäßnennen, einen Link zur Creative Commons Lizenz beifügen und angeben, ob Änderungen vorgenommen wurden.

\section{Literatur}

1. Bedi A, Dines J, Warren RF, Dines DM (2010) Massive tears of the rotator cuff. J Bone Joint Surg Am 92:1894-1908

2. Burkhart SS, Nottage WM, Ogilvie-Harris DJ, Kohn HS, Pachelli A (1994) Partial repair of irreparable rotator cuff tears. Arthroscopy 10:363-370

3. Chung SW, Kim JY, Kim MH, Kim SH, Oh JH (2013) Arthroscopic repair of massive rotator cuff tears: Outcome and analysis of factors associated with healing failure or poor postoperative function. Am J Sports Med 41:1674-1683

4. Collin P, Matsumura N, Ladermann A, Denard PJ, Walch $G$ (2014) Relationship between massive chronic rotator cuff tear pattern and loss of active shoulder range of motion. J Shoulder Elbow Surg 23:1195-1202
5. Denard PJ, Brady PC, Adams CR, Tokish JM, Burkhart SS (2018) Preliminary results of arthroscopic superior capsule reconstruction with dermal allograft. Arthroscopy 34:93-99

6. DeOrio JK, Cofield RH (1984) Results of a second attempt at surgical repair of a failed initial rotatorcuff repair. JBone Joint Surg Am 66:563-567

7. Dwyer T, Razmjou H, Henry P, Gosselin-Fournier S, Holtby R (2015) Association between preoperative magnetic resonance imaging and reparability of large and massive rotator cuff tears. Knee Surg Sports Traumatol Arthrosc 23:415-422

8. Galatz LM, Ball CM, Teefey SA, Middleton WD, Yamaguchi K (2004) The outcome and repair integrity of completely arthroscopically repaired large and massive rotator cuff tears. J Bone Joint Surg Am 86-a:219-224

9. Gerber C, Fuchs B, Hodler J (2000) The results of repair of massive tears of the rotator cuff. J Bone Joint Surg Am 82:505-515

10. Gerber C, Vinh TS, Hertel R, Hess CW (1988) Latissimus dorsi transfer for the treatment of massive tears of the rotator cuff. A preliminary report. Clin Orthop Relat Res 232:51-61

11. Goutallier D, Postel JM, Bernageau J, Lavau L, Voisin MC (1994) Fatty muscle degeneration in cuff ruptures. Pre- and postoperative evaluation by $\mathrm{CT}$ scan. Clin Orthop Relat Res 304:78-83

12. Grammont PM, Baulot E (1993) Delta shoulder prosthesis for rotator cuff rupture. Orthopedics 16:65-68

13. Hamada K, Fukuda H, Mikasa M, Kobayashi Y (1990) Roentgenographic findings in massive rotator cuff tears. A long-term observation. Clin Orthop Relat Res 254:92-96

14. Heuberer PR, Kolblinger R, Buchleitner $S$, Pauzenberger L, Laky B, Auffarth A, Moroder P, Salem S, Kriegleder B, Anderl W (2016) Arthroscopic management of massive rotator cuff tears: an evaluation of debridement, complete, and partial repair with and without force couple restoration. Knee Surg Sports Traumatol Arthrosc 24:3828-3837

15. Hirahara AM, Adams CR (2015) Arthroscopic superior capsular reconstruction for treatment of massive irreparable rotator cuff tears. Arthrosc Tech 4:e637-e641

16. Hirahara AM, Andersen WJ, Panero AJ (2017) Superior capsular reconstruction: Clinical outcomes after minimum 2-year follow-up. Am J Orthop (Belle Mead, NJ) 46:266-278

17. Lee SJ, Min YK (2018) Can inadequate acromiohumeral distance improvement and poor posterior remnant tissue be the predictive factors of re-tear? Preliminary outcomes of arthroscopic superior capsular reconstruction. Knee Surg Sports Traumatol Arthrosc 26(7):2205-2213. https://doi.org/10. 1007/s00167-018-4912-8

18. Mihata T, Bui CNH, Akeda M, Cavagnaro MA, Kuenzler M, Peterson AB, McGarry MH, Itami Y, Limpisvasti O, Neo M, Lee TQ (2017) A biomechanical cadaveric study comparing superior capsule reconstruction using fascia lata allograft with human dermal allograft for irreparable rotator cuff tear. JShoulder Elbow Surg 26:2158-2166

19. Mihata $T$, Lee TQ, Fukunishi K, Itami Y, Fujisawa Y, Kawakami T, Ohue M, Neo M (2018) Return to sports and physical work after arthroscopic superior capsule reconstruction among patients with irreparable rotator cuff tears. Am JSports Med 46:1077-1083

20. Mihata T, Lee TQ, Watanabe C, Fukunishi K, Ohue M, Tsujimura T, Kinoshita M (2013) Clinical results of arthroscopic superior capsule reconstruction 
for irreparable rotator cuff tears. Arthroscopy 29:459-470

21. Mihata T, McGarry MH, Kahn T, Goldberg I, Neo M, Lee TQ (2016) Biomechanical effect of thickness and tension of fascia Lata graft on Glenohumeral stability for superior capsule reconstruction in irreparable Supraspinatus tears. Arthroscopy 32:418-426

22. Mihata T, McGarry MH, Kahn T, Goldberg I, Neo M, Lee TQ (2016) Biomechanical effects of Acromioplasty on superior capsule reconstruction for irreparable Supraspinatus tendon tears. Am J Sports Med 44:191-197

23. Mihata T, McGarry MH, Kahn T, Goldberg I, Neo M, Lee TQ (2016) Biomechanical role of capsular continuity in superior capsule reconstruction for irreparable tears of the Supraspinatus tendon. Am J Sports Med 44:1423-1430

24. Mihata T, McGarry MH, Pirolo JM, Kinoshita M, Lee TQ (2012) Superior capsule reconstruction to restore superior stability in irreparable rotator cuff tears: A biomechanical cadaveric study. Am J Sports Med 40:2248-2255

25. Minkus M, Scheibel M (2017) Superior Capsular Reconstruction (SCR). Obere Extremität 12:51-53

26. Nimura A, Kato A, Yamaguchi K, Mochizuki T, Okawa A, Sugaya H, Akita K (2012) The superior capsule of the shoulder joint complements the insertion of the rotator cuff. J Shoulder Elbow Surg 21:867-872

27. Pennington WT, Bartz BA, Pauli JM, Walker CE, Schmidt W (2018) Arthroscopic superior capsular reconstruction with acellular dermal allograft for the treatment of massive irreparable rotato cuff tears: Short-term clinical outcomes and the radiographic parameter of superior capsular distance. Arthroscopy 34(6):1764-1773. https:// doi.org/10.1016/j.arthro.2018.01.009

28. Plachel F, Moroder P, Gerhardt C, Scheibel M (2017) Anterosuperiore Rotatorenmanschettenläsion beim jungen Patienten. Arthroskopie 30:216-222

29. Resch H, Povacz P, Ritter E, Matschi W (2000) Transfer of the pectoralis major muscle for the treatment of irreparable rupture of the subscapularis tendon. J Bone Joint Surg Am 82:372-382

30. Savarese E, Romeo R (2012) New solution for massive, irreparable rotator cuff tears: the subacromial "biodegradable spacer". Arthrosc Tech 1:e69-74

31. Shon MS, Koh KH, Lim TK, Kim WJ, Kim KC, Yoo JC (2015) Arthroscopic partial repair of irreparable rotator cuff tears: Preoperative factors associated with outcome deterioration over 2 years. Am J Sports Med 43:1965-1975

32. Walch G, Edwards TB, Boulahia A, Nove-Josserand L, Neyton L, Szabo I (2005) Arthroscopic tenotomy of the long head of the biceps in the treatment of rotator cuff tears: Clinical and radiographic results of 307 cases. JShoulder Elbow Surg 14:238-246

33. Werner CM, Conrad SJ, Meyer DC, Keller A, Hodler J, Gerber C (2008) Intermethod agreement and interobserver correlation of radiologic acromiohumeral distance measurements. J Shoulder Elbow Surg 17:237-240 